\title{
Active control of compression systems using drive torque; a backstepping approach
}

\author{
Bjørnar Bøhagen and Jan Tommy Gravdahl \\ Department of Engineering Cybernetics, Norwegian University of Science and Technology \\ N-7491 Trondheim, NORWAY \\ Email: \{bjornar.bohagen,tommy.gravdahl\}@itk.ntnu.no
}

\begin{abstract}
A novel control law for a compression system using the drive torque as control input is presented. Backstepping is used in the derivation of the control law, which does not require the explicit knowledge of a compressor model. The only information required from the compressor is an upper bound on the ratio of growth rate in compressor mass flow and speed.
\end{abstract}

\section{INTRODUCTION}

Towards low mass flows, the stable operating region of centrifugal compressors is bounded due to the occurrence of surge. This phenomenon is characterized by oscillations in system states such as pressure and mass flow, and is undesirable since it introduces the possibility of severe damage to the machine due to vibrations and high thermal loading resulting from lowered efficiency.

Compressor performance is usual described with a compressor map, Fig. 1. This map describes the relation of compressor pressure ratio, mass flow and speed using constant speed lines in a flow-pressure coordinate system. Surge is considered as an unstable operational mode of the compressor and the stability boundary in the compressor map is called the surge line. This line divides the compressor map in two regions, where the region to the left and right of the surge line corresponds to stable and unstable regions respectively.

Traditionally, surge has been avoided using surge avoidance schemes. Such schemes use various means in order to keep the operating point of the compressor away from the region where surge occurs. Typically, a surge control line is drawn at a distance away from the surge line, leaving a surge margin in the compressor map, and the surge avoidance scheme ensures that the operating point does not cross this line, Fig. 1. This method restricts the operating range of the machine to the region in which the system is open loop stable, and efficiency is limited. Usually a recycle line around the compressor is used for actuation.

Active surge control is fundamentally different from surge avoidance. In an active surge control scheme the open loop unstable region of the compressor map is sought stabilized with feedback rather than avoided. Thus, the operating regime of the machine is enlarged. Active surge control of compressors was first introduced by [1], and since then a number of results have been published. Different actuators have been used and examples include recycle, bleed and throttle valves, gas injection, variable guide vanes and drive torque. For an overview, consult [2] and [3].

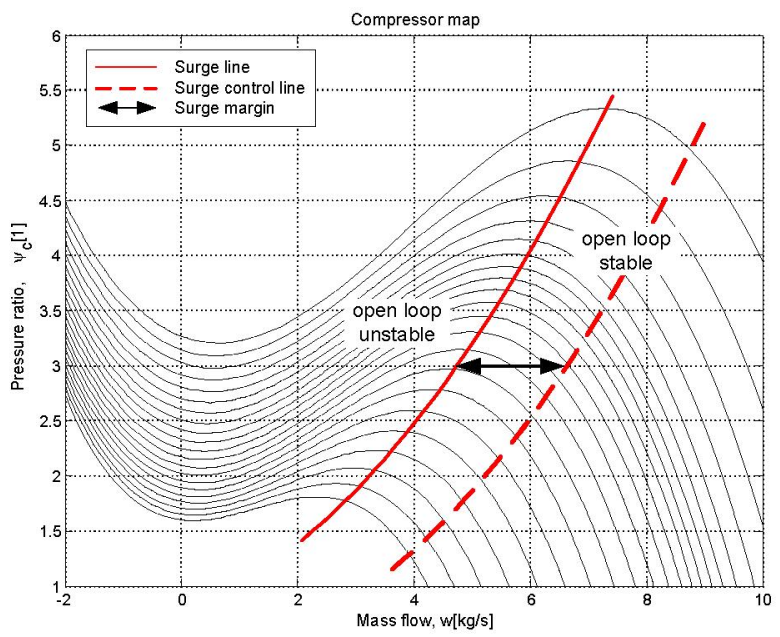

Fig. 1. Compressor performance and surge related definitions

In this paper we investigate the possibility of using only the drive torque to actively stabilize the compression system. The idea was initially introduced in [4], where it was shown that by using the compressor speed as input, the system could be stabilized. Furthermore, the authors showed convergence towards a desired equilibrium if the speed dynamics was included and the drive torque was used as input.

\section{MODEL}

A classical result in the field of compressor surge modeling is the model of Greitzer [5] who modelled a basic compression system consisting of a compressor, a plenum volume, in-between ducting and a throttle valve as shown in Fig. 2. The authors of [6] extended the Greizer-model to include rotational speed as a state in the model. A similar model was derived in [7] using an approach based on energy analysis.

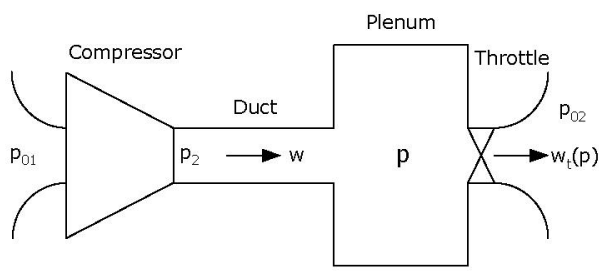

Fig. 2. Compression system 
The model is derived by calculating the mass balance of the plenum volume, integrating the one dimensional Euler equation (the momentum balance) over the length of the duct and calculating the torque balance of the rotating shaft. This gives the model

$$
\begin{aligned}
\dot{p} & =\frac{a_{01}^{2}}{V_{p}}\left(w-w_{t}(p)\right) \\
\dot{w} & =\frac{A_{1}}{L_{c}}\left(p_{2}(w, \omega)-p\right) \\
\dot{\omega} & =\frac{1}{J}\left(\tau_{d}-\tau_{c}(w, \omega)\right)
\end{aligned}
$$

where $p$ is the plenum pressure, $w$ is the compressor mass flow, $\omega$ is the rotational speed of the shaft driving the compressor, $w_{t}(p)$ is the mass flow through the throttle valve, $p_{2}(w, \omega)$ is the pressure downstream the compressor, $\tau_{d}$ is the torque driving the compressor, $\tau_{c}(w, \omega)$ is the compressor load torque, $a_{01}$ is the sonic velocity at ambient conditions, $V_{p}$ is the plenum volume, $A_{1}$ is the duct throughflow area, $L_{c}$ is the length of the duct and $J$ is the inertia of rotating parts. The mass flow through the throttle and the compressor torque are modelled as

$$
\begin{aligned}
w_{t}(p) & =k_{t} \operatorname{sign}\left(p-p_{02}\right) \sqrt{\left|p-p_{02}\right|} \\
\tau_{c}(w, \omega) & =\sigma r_{2}^{2}|w| \omega
\end{aligned}
$$

where $k_{t}>0$ is a parameter proportional to the throttle opening, $p_{02}$ is the ambient pressure downstream of the throttle, $\sigma$ is the slip factor and $r_{2}$ is the impeller diameter. The model of throttle valve mass flow is slightly modified relative to earlier modelling. The modification involves including the possibility of negative mass flow through the valve, and to this end the throttle characteristic is assumed to be symmetric.

Pressure downstream of the compressor is often expressed using the compressor characteristics $\psi(w, \omega)$, where $p_{2}(w, \omega)=\psi(w, \omega) p_{01}$ and $p_{01}$ is the ambient pressure upstream of the compressor. A typical compressor characteristic is illustrated in Fig. 1. For a more detailed model of the compressor characteristic the reader is referred to [7].

Due to application of Lyapunov theory the system states are shifted such that the equilibrium of interest is represented by the origin in a new coordinate system. Let the equilibrium of interest be denoted by $(\cdot)^{0}$. From (1)-(3) the relations

$$
\begin{aligned}
w^{0} & =w_{t}\left(p^{0}\right) \\
p_{2}\left(w^{0}, \omega^{0}\right) & =p^{0} \\
\tau_{d}^{0} & =\tau_{c}\left(w^{0}, \omega^{0}\right)
\end{aligned}
$$

are found for system equilibrium.

Definition 1: Error variables

$$
\begin{aligned}
x_{1} & =p-p^{0} \\
x_{2} & =w-w^{0} \\
x_{3} & =\omega-\omega^{0} \\
u & =\tau_{d}-\tau_{d}^{0}
\end{aligned}
$$

With Definition 1, (1)-(3) and (6)-(8) the equilibrium is shifted to the origin

$$
\begin{aligned}
& \dot{x}_{1}=k_{1}\left(x_{2}-f_{1}\left(x_{1}\right)\right) \\
& \dot{x}_{2}=k_{2}\left(f_{2}\left(x_{2}, x_{3}\right)-x_{1}\right) \\
& \dot{x}_{3}=k_{3}\left(u+f_{3}\left(x_{2}, x_{3}\right)\right)
\end{aligned}
$$

where

$$
\begin{aligned}
f_{1}\left(x_{1}\right) & =w^{0}-w_{t}\left(x_{1}+p^{0}\right) \\
f_{2}\left(x_{2}, x_{3}\right) & =p_{2}\left(x_{2}+w^{0}, x_{3}+\omega^{0}\right)-p^{0} \\
f_{3}\left(x_{2}, x_{3}\right) & =\tau_{d}^{0}-\tau_{c}\left(x_{2}+w^{0}, x_{3}+\omega^{0}\right)
\end{aligned}
$$

and $k_{1}=\frac{a_{01}^{2}}{V_{p}}, k_{2}=\frac{A_{1}}{L_{c}}$ and $k_{3}=\frac{1}{J}$ are all positive constants. The following properties can be recognized for $f_{1}, f_{2}$ and $f_{3}$

$$
\begin{aligned}
f_{1}(0)=f_{2}(0,0)=f_{3}(0,0) & =0 \\
(a-b)\left(f_{1}(a)-f_{1}(b)\right) & <0 \forall a \neq b \\
(a-b)\left(f_{2}\left(x_{2}, a\right)-f_{2}\left(x_{2}, b\right)\right) & >0 \forall a \neq b \\
(a-b)\left(f_{3}\left(x_{2}, a\right)-f_{3}\left(x_{2}, b\right)\right) & \leq 0 \forall a \neq b
\end{aligned}
$$

where the fact that $\frac{\partial p_{2}(w, \omega)}{\partial \omega}>0$ has been used. This is a property of the compressor.

\section{STABILIZATION VIA BACKSTEPPING}

In this section backstepping is applied to design a input $u$ to asymptotically stabilize the origin of (9)-(11). By a closer look at the model it is clear that it is in a pure-feedback form [8]. The only reason for it not to be in a strict-feedback form lies in the $x_{2}$ dynamics where $x_{3}$ does not appear affine, but through the function $f_{2}$. The practical implication of this is that stepping back on $x_{3}$ becomes more complicated, especially in this case since the design is done without the explicit knowledge of the function $f_{2}\left(x_{2}, x_{3}\right)$.

Using backstepping to stabilize the compressions system was also done in [9], where flow through the throttle was used as control input.

The first step consists of stabilizing $x_{1}$ using $x_{2}$ as virtual control. Define

$$
z_{1}=x_{1}
$$

which by using (9) gives

$$
\dot{z}_{1}=k_{1}\left(x_{2}+f_{1}\left(z_{1}\right)\right)
$$

where $x_{2}$ is considered as virtual control. Define next

$$
z_{2}=x_{2}-\alpha_{1}\left(z_{1}\right)
$$

which describes the difference between $x_{2}$ and its desired expression $\alpha_{1}\left(z_{1}\right)$ to be chosen. Consider now the function

$$
V_{1}\left(z_{1}\right)=\frac{1}{2} d_{1} z_{1}^{2}
$$

where $d_{1}$ is a positive constant. Using (21), the time derivative of $V_{1}$ along the solution of (20) is found as

$$
\dot{V}_{1}\left(z_{1}\right)=d_{1} k_{1} z_{1} \alpha_{1}\left(z_{1}\right)+d_{1} k_{1} z_{1} f_{1}\left(z_{1}\right)+d_{1} k_{1} z_{1} z_{2}
$$

where $\alpha_{1}\left(z_{1}\right)$ is at our disposal to render $\dot{V}_{1}$ negative definite in $z_{1}$ and the term $z_{1} z_{2}$ is dealt with in the next step since it contains $z_{2}$. From (16) it can be recognized that the term 
$z_{1} f_{1}\left(z_{1}\right)$ is negative definite, which implies that $\alpha_{1}\left(z_{1}\right)$ is not needed to render $\dot{V}_{1}$ negative definite in $z_{1}$. This motivates the choice

$$
\alpha_{1}\left(z_{1}\right)=0
$$

resulting in

$$
\dot{V}_{1}\left(z_{1}\right)=d_{1} k_{1} z_{1} f_{1}\left(z_{1}\right)+d_{1} k_{1} z_{1} z_{2}
$$

The second step consists of stabilizing $z_{2}$ using $x_{3}$ as virtual control. Using (21), (10), (24) and (19), the $z_{2}$ dynamics is found as

$$
\dot{z}_{2}=k_{2}\left(f_{2}\left(z_{2}, x_{3}\right)-z_{1}\right)
$$

where $x_{3}$ is considered as virtual control. Define

$$
z_{3}=x_{3}-\alpha_{2}\left(z_{1}, z_{2}\right)
$$

which describes the difference between $x_{3}$ and the desired expression $\alpha_{2}\left(z_{1}, z_{2}\right)$ to be chosen. Consider now the function

$$
V_{2}\left(z_{1}, z_{2}\right)=V_{1}\left(z_{1}\right)+\frac{1}{2} d_{2} z_{2}^{2}
$$

where $d_{2}$ is a positive constant. Using (25), (26) and (27) the time derivative of $V_{2}$ is found as

$$
\begin{aligned}
\dot{V}_{2}\left(z_{1}, z_{2}\right)= & d_{1} k_{1} z_{1} f_{1}\left(z_{1}\right)+d_{2} k_{2} z_{2} f_{2}\left(z_{2}, \alpha_{2}\left(z_{1}, z_{2}\right)\right) \\
& +d_{2}\left(\frac{d_{1}}{d_{2}} k_{1}-k_{2}\right) z_{1} z_{2} \\
& +d_{2} k_{2} z_{2} \bar{f}_{2}\left(z_{1}, z_{2}, z_{3}\right)
\end{aligned}
$$

where

$\bar{f}_{2}\left(z_{1}, z_{2}, z_{3}\right)=f_{2}\left(z_{2}, z_{3}+\alpha_{2}\left(z_{1}, z_{2}\right)\right)-f_{2}\left(z_{2}, \alpha_{2}\left(z_{1}, z_{2}\right)\right)$

In (29) $\alpha_{2}\left(z_{1}, z_{2}\right)$ is at our disposal to render the term $z_{2} f_{2}\left(z_{2}, \alpha_{2}\left(z_{1}, z_{2}\right)\right)$ negative definite in $z_{2}$, the term $z_{1} z_{2}$ can be cancelled by a appropriate choice of $d_{1}$ and the term $z_{2} \bar{f}_{2}\left(z_{1}, z_{2}, z_{3}\right)$ is dealt with in the next step since it contains $z_{3}$. By requiring $\left.f_{2}\left(z_{2}, \alpha_{2}\left(z_{1}, z_{2}\right)\right)\right|_{z_{2}=0}=0$ and $\frac{d f_{2}\left(z_{2}, \alpha_{2}\left(z_{1}, z_{2}\right)\right)}{d z_{2}}<0$ the function $f_{2}\left(z_{2}, \alpha_{2}\left(z_{1}, z_{2}\right)\right)$ is forced to lie strictly in the 2 . and 4 . quadrant of the $\left(z_{2}, f_{2}\left(z_{2}, \alpha_{2}\left(z_{1}, z_{2}\right)\right)\right)$-coordinate system, from which it follows that $z_{2} f_{2}\left(z_{2}, \alpha_{2}\left(z_{1}, z_{2}\right)\right)$ is negative definite in $z_{2}$. Moreover, if the inequality is strengthened to $\frac{d f_{2}\left(z_{2}, \alpha_{2}\left(z_{1}, z_{2}\right)\right)}{d z_{2}} \leq-\delta_{2}$ for some positive constant $\delta_{2}$, the upper bound $z_{2} f_{2}\left(z_{2}, \alpha_{2}\left(z_{1}, z_{2}\right)\right) \leq-\delta_{2} z_{2}^{2}$ is achieved. This approach was taken in [4] where

$$
\alpha_{2}\left(z_{1}, z_{2}\right)=-c_{2} z_{2}
$$

was used along with the requirement

$$
c_{2} \geq\left.\frac{\frac{\partial f_{2}\left(r_{1}, r_{2}\right)}{\partial r_{1}}+\delta_{2}}{\frac{\partial f_{2}\left(r_{1}, r_{2}\right)}{\partial r_{2}}}\right|_{\left(r_{1}, r_{2}\right)=\left(z_{2},-c_{2} z_{2}\right)}
$$

Combining (31) and (32) with the choice $d_{1}=\frac{d_{2}}{k_{1}} k_{2}$, an upper bound on (29) is given by

$$
\begin{aligned}
\dot{V}_{2}\left(z_{1}, z_{2}\right) \leq & d_{2} k_{2} z_{1} f_{1}\left(z_{1}\right)-d_{2} k_{2} \delta_{2} z_{2}^{2} \\
& +d_{2} k_{2} z_{2} \bar{f}_{2}\left(z_{1}, z_{2}, z_{3}\right)
\end{aligned}
$$

The third and final step consists of stabilizing $z_{3}$ with the control input $u$. Using (27), (11), (31) and (26) the $z_{3}$ dynamics is found as

$$
\begin{aligned}
\dot{z}_{3}= & k_{3} u+k_{3} f_{3}\left(z_{2}, z_{3}-c_{2} z_{2}\right) \\
& +c_{2} k_{2} f_{2}\left(x_{2}, z_{2}, z_{3}-c_{2} z_{2}\right)-c_{2} k_{2} z_{1}
\end{aligned}
$$

Consider now the function

$$
V_{3}\left(z_{1}, z_{2}, z_{3}\right)=V_{2}\left(z_{1}, z_{2}\right)+\frac{1}{2} d_{3} z_{3}^{2}
$$

where $d_{3}$ is a positive constant. Using (33) and (34), the time derivative of $V_{3}$ is found as

$$
\begin{aligned}
\dot{V}_{3}\left(z_{1}, z_{2}, z_{3}\right) \leq & d_{2} k_{2} z_{1} f_{1}\left(z_{1}\right)-d_{2} k_{2} \delta_{2} z_{2}^{2} \\
& +d_{2} k_{2} z_{2} \bar{f}_{2}\left(z_{1}, z_{2}, z_{3}\right) \\
& +d_{3} k_{3} z_{3}\left(\begin{array}{c}
u+f_{3}\left(x_{2}, x_{3}\right) \\
+\frac{c_{2} k_{2}}{k_{3}} f_{2}\left(x_{2}, x_{3}\right) \\
-\frac{c_{2} k_{2}}{k_{3}} x_{1}
\end{array}\right)
\end{aligned}
$$

where $u$ is at our disposal to render $\dot{V}_{2}$ negative definite in $\left(z_{1}, z_{2}, z_{3}\right)$. Before choosing the input $u$, we seek an upper bound of the term $z_{2} \bar{f}_{2}\left(z_{1}, z_{2}, z_{3}\right)$. Using the mean value theorem on (30) and then Young's inequality ${ }^{1}$, it can be recognized that

$$
\begin{aligned}
z_{2} \bar{f}_{2}\left(z_{1}, z_{2}, z_{3}\right) & =\frac{\partial f_{2}\left(r_{3}, r_{4}\right)}{\partial r_{4}} z_{2} z_{3} \\
& \leq \frac{1}{2} \frac{\partial f_{2}\left(r_{3}, r_{4}\right)}{\partial r_{4}}\left(\gamma_{2} z_{2}^{2}+\frac{1}{\gamma_{2}} z_{3}^{2}\right)
\end{aligned}
$$

where $\left(r_{3}, r_{4}\right) \in\left(z_{2}, L_{1}\right)$ and $L_{1}$ is the line segment joining $z_{3}+\alpha_{2}\left(z_{1}, z_{2}\right)$ and $\alpha_{2}\left(z_{1}, z_{2}\right)$. This suggests that the input $u$ should leave a term which is quadratic in $z_{3}$ in order to handle the corresponding term resulting from the bound on $z_{2} \bar{f}_{2}\left(z_{1}, z_{2}, z_{3}\right)$. Consider the control law

$$
u=-f_{3}\left(x_{2}, x_{3}\right)-\frac{c_{2} k_{2}}{k_{3}} f_{2}\left(x_{2}, x_{3}\right)+\frac{c_{2} k_{2}}{k_{3}} x_{1}-c_{3} z_{3}
$$

which can be recognized to include three canceling terms in addition to the stabilizing term $c_{3} z_{3}$. The terms cancelled involves compressor torque, pressure downstream the compressor and plenum pressure, whereas the stabilizing term involves a linear feedback combination of mass flow and compressor velocity. Using (37) and (38) in (36) results in

$$
\dot{V}_{3}\left(z_{1}, z_{2}, z_{3}\right) \leq d_{2} k_{2} z_{1} f_{1}\left(z_{1}\right)-q_{2} z_{2}^{2}-q_{3} z_{3}^{2}
$$

where

$$
\begin{aligned}
q_{2} & =d_{2} k_{2}\left(\delta_{2}-\frac{1}{2} \gamma_{2} \frac{\partial f_{2}\left(r_{3}, r_{4}\right)}{\partial r_{4}}\right) \\
q_{3} & =d_{3} k_{3}\left(c_{3}-\frac{d_{2}}{d_{3}} \frac{k_{2}}{2 k_{3} \gamma_{2}} \frac{\partial f_{2}\left(r_{3}, r_{4}\right)}{\partial r_{4}}\right)
\end{aligned}
$$

The parameters $q_{2}$ and $q_{3}$ can be made positive by choosing $\gamma_{2}$ small enough so that it suppresses the amplitude of $\frac{1}{2} \gamma_{2} \frac{\partial f_{2}\left(r_{3}, r_{4}\right)}{\partial r_{4}}$ sufficiently relative to $\delta_{2}$ and choosing $\frac{d_{2}}{d_{3}}$ small enough so that it suppresses the amplitude of

\footnotetext{
${ }^{1}$ In its simplest form it states $\left|r_{1}\right|\left|r_{2}\right| \leq \frac{1}{2}\left(\gamma r_{1}^{2}+\frac{1}{\gamma} r_{2}^{2}\right)$ where $\gamma>0$
} 
$\frac{k_{2}}{2 k_{3} \gamma_{2}} \frac{\partial f_{2}\left(r_{3}, r_{4}\right)}{\partial r_{4}}$ relative to $c_{3}$. To formalize the result, define $D_{z}^{1}=\left\{z \in R^{3}|| z_{2}\left|\leq z_{2, \max },\right| z_{3} \mid \leq z_{3, \max }\right\}$ where $z_{2, \max }$ and $z_{3, \max }$ are arbitrarily large positive constants. Assume that $\frac{\partial p_{2}(w, \omega)}{\partial \omega}$ is bounded for bounded $(w, \omega)$. From (35), (39)-(41) and appropriate choice of $\gamma_{2}$ and $\frac{d_{2}}{d_{3}}$ it can be recognized that $V_{3}$ is positive definite on $R^{3}$ and $\dot{V}_{3}$ is negative definite on $D_{z}^{1}$, by which it is concluded that the origin of the $z$ system is semi globally asymptotically stable. Moreover, if $\frac{\partial p_{2}(w, \omega)}{\partial \omega}$ is globally bounded in $(w, \omega)$ the result holds globally. The preceding analysis is summarized in a proposition.

Proposition 2: Assume that $\frac{\partial p_{2}(w, \omega)}{\partial \omega}$ is bounded for bounded $(w, \omega)$ and $f_{1}\left(x_{1}\right)$ satisfy $(16)$. Then the control law

$u=-f_{3}\left(x_{2}, x_{3}\right)-\frac{c_{2} k_{2}}{k_{3}} f_{2}\left(x_{2}, x_{3}\right)+\frac{c_{2} k_{2}}{k_{3}} x_{1}-c_{2} c_{3} x_{2}-c_{3} x_{3}$ where

$$
c_{2} \geq\left.\frac{\frac{\partial f_{2}\left(r_{1}, r_{2}\right)}{\partial r_{1}}+\delta_{2}}{\frac{\partial f_{2}\left(r_{1}, r_{2}\right)}{\partial r_{2}}}\right|_{\left(r_{1}, r_{2}\right)=\left(x_{2},-c_{2} x_{2}\right)}
$$

and $c_{3}>0$ makes the origin of (9)-(11) semi globally asymptotically stable. Moreover, if $\frac{\partial p_{2}(w, \omega)}{\partial \omega}$ is globally bounded in $(w, \omega)$ the origin is globally asymptotically stable.

By using the throttle valve model (4) it can be recognized that

$$
z_{1} f_{1}\left(z_{1}\right) \leq-\delta_{1} z_{1}^{2} \forall\left\{\left|z_{1}\right| \leq z_{1, \max }\right\}
$$

where $\delta_{1}$ is a positive constant and $z_{1, \min }$ and $z_{1, \max }$ are arbitrarily large positive constants. Using (42) in (39) an upper bound on $\dot{V}_{3}$ is given by

$$
\dot{V}_{3}\left(z_{1}, z_{2}, z_{3}\right) \leq-q_{1} z_{1}^{2}-q_{2} z_{2}^{2}-q_{3} z_{3}^{2} \forall z \in D_{z}^{2}
$$

where $q_{1}=\delta_{1} d_{2} k_{2}$ is positive and $D_{z}^{2}=$ $\left\{z \in R^{3}|| z_{1}\left|\leq z_{1, \max },\right| z_{2}\left|\leq z_{2, \max },\right| z_{3} \mid \leq z_{3, \max }\right\}$.

The introduction of $D_{z}^{2}$ poses no new limitation on the stability results since the introduction of $D_{z}^{1}$ already have limited the results to be semi global. From (35) and (43) semi global exponential stability of the origin of $z$ is concluded. An upper bound on the solution is given by [10]

$$
\|z(t)\|_{2} \leq k_{z}\left\|z\left(t_{0}\right)\right\|_{2} e^{-\lambda_{z}\left(t-t_{0}\right)} \forall\left\|z\left(t_{0}\right)\right\|_{2} \leq r_{z}
$$

where $k_{z}=\sqrt{\frac{\max \left\{p_{i}\right\}}{\min \left\{p_{i}\right\}}}, \lambda_{z}=\frac{\min \left\{q_{i}\right\}}{\max \left\{p_{i}\right\}}$ and $r_{z}$ is an arbitrarily large constant. Moreover, the map from $x$ to $z$ is linear and given by $z=T x$ where

$$
T=\left[\begin{array}{ccc}
1 & 0 & 0 \\
0 & 1 & 0 \\
0 & c_{2} & 1
\end{array}\right]
$$

is nonsingular, which guarantees the existence of $x=T^{-1} z$. An upper bound on $x(t)$ can now be calculated

$$
\|x(t)\|_{2} \leq k_{x}\left\|x\left(t_{0}\right)\right\|_{2} e^{-\lambda_{z}\left(t-t_{0}\right)} \forall\left\|x\left(t_{0}\right)\right\|_{2} \leq r_{x}
$$

where $k_{x}=k_{z}\left\|T^{-1}\right\|_{2}^{2}$ and $r_{x}=\left\|T^{-1}\right\|_{2} r_{z}$ is an arbitrarily large constant. The exponential result is summarized in Corollary 3

Corollary 3: The control law from Proposition 2 makes the origin of (9)-(11) semi globally exponentially stable.

The difference of Proposition 2 and Corollary 3 lies in the explicit use of (4) in the corollary rather than its property.

\section{A. Avoiding cancellation of compressor torque}

Suppose that compensating for the compressor torque in the control law for some reason is undesirable. This can for instance be due to the lack of this measurement or measurements needed in a model of the torque, or simply that the quality of the model is poor in some region of operation.

Notice that replacing $f_{3}\left(x_{2}, x_{3}\right)$ with $f_{3}\left(x_{2}, \alpha_{1}\left(x_{2}\right)\right)$ in the control law (38) and applying (18) gives the same upper bound on $\dot{V}_{3}$ as found for (39) or (43). Wether or not this is a good replacement depends on the regions in which $x_{3}$ and $-c_{1} x_{2}$ is expected to operate relative to the regions for which $f_{3}$ is expected to be a better approximation of the compressor torque in its second argument.

Consider now the control law without the cancellation of compressor torque

$$
u=-\frac{c_{2} k_{2}}{k_{3}} f_{2}\left(x_{2}, x_{3}\right)+\frac{c_{2} k_{2}}{k_{3}} x_{1}-c_{2} c_{3} x_{2}-c_{3} x_{3}
$$

Using (44) in (36) gives

$\dot{V}_{3}\left(z_{1}, z_{2}, z_{3}\right) \leq d_{2} k_{2} z_{1} f_{1}\left(z_{1}\right)-q_{2} z_{2}^{2}-q_{3} z_{3}^{2}+d_{3} k_{3} z_{3} f_{3}\left(z_{2}, x_{3}\right)$

where the term $z_{3} f_{3}\left(x_{2}, x_{3}\right)$ must be further analyzed. The approach taken is to upper bound this term in quadratic terms of $z_{2}$ and $z_{3}$. The first step towards this upper bound is to use (27) and (18) to rewrite the term as

$$
\begin{aligned}
z_{3} f_{3}\left(z_{2}, x_{3}\right)= & z_{3}\left(f_{3}\left(z_{2}, x_{3}\right)-f_{3}\left(z_{2}, \alpha_{2}\left(z_{2}\right)\right)\right) \\
& +z_{3} f_{3}\left(z_{2}, \alpha_{2}\left(z_{2}\right)\right) \\
\leq & z_{3} f_{3}\left(z_{2}, \alpha_{2}\left(z_{2}\right)\right)
\end{aligned}
$$

Next the function $f_{3}\left(z_{2}, \alpha_{2}\left(z_{2}\right)\right)$ is rewritten using (14), (5), (31), (21), (24) and Definition 1

$$
f_{3}\left(z_{2}, \alpha_{2}\left(z_{2}\right)\right)=c_{2} \sigma r_{2}^{2}|w| z_{2}-\left(\sigma r_{2}^{2} \omega^{0}\left|z_{2}+w^{0}\right|-\tau_{d}^{0}\right)
$$

where the mass flow, $w$, now is considered as a time varying signal. Finally, (47), (8) and Young's inequality are used to upper bound $z_{3} f_{3}\left(z_{2}, \alpha_{2}\left(z_{2}\right)\right)$ as

$$
\begin{aligned}
z_{3} f_{3}\left(z_{2}, \alpha_{2}\left(z_{2}\right)\right) \leq & c_{2} \sigma r_{2}^{2}|w|\left|z_{2}\right|\left|z_{3}\right| \\
& +\left|z_{3}\right|\left|\left(\sigma r_{2}^{2} \omega^{0}\left|z_{2}\right|+\sigma r_{2}^{2} \omega^{0}\left|w^{0}\right|-\tau_{d}^{0}\right)\right| \\
= & \left(c_{2} \sigma r_{2}^{2}|w|+\sigma r_{2}^{2} \omega^{0}\right)\left|z_{2}\right|\left|z_{3}\right| \\
\leq & \gamma_{3} \sigma r_{2}^{2} \frac{1}{2}\left(c_{2}|w|+\omega^{0}\right) z_{2}^{2} \\
& +\frac{1}{\gamma_{3}} \frac{1}{2} \sigma r_{2}^{2}\left(c_{2}|w|+\omega^{0}\right) z_{3}^{2}
\end{aligned}
$$

Using (46) and (48) in (45) results in where

$$
\dot{V}_{3}\left(z_{1}, z_{2}, z_{3}\right) \leq d_{2} k_{2} z_{1} f_{1}\left(z_{1}\right)-q_{2}^{\prime} z_{2}^{2}-q_{3}^{\prime} z_{3}^{2}
$$

$$
\begin{aligned}
& q_{2}^{\prime}=d_{2} k_{2}\left(\begin{array}{c}
\delta_{2}-\gamma_{2} \frac{1}{2} \frac{\partial f_{2}\left(r_{3}, r_{4}\right)}{\partial r_{4}} \\
-\gamma_{3} \frac{d_{3}}{d_{2}} \frac{k_{3} \sigma r_{2}^{2}}{2 k_{2}}\left(c_{2}|w|+\omega^{0}\right)
\end{array}\right) \\
& q_{3}^{\prime}=d_{3} k_{3}\left(\begin{array}{c}
c_{3}-\frac{d_{2}}{d_{3}} \frac{k_{2}}{2 k_{3} \gamma_{2}} \frac{\partial f_{2}\left(r_{3}, r_{4}\right)}{\partial r_{4}} \\
-\frac{1}{\gamma_{3}} \frac{\sigma r_{2}^{2}}{2}\left(c_{2}|w|+\omega^{0}\right)
\end{array}\right)
\end{aligned}
$$

Following the same arguments as in the case where com- 
pressor torque was included in the control law, $q_{2}^{\prime}$ is made positive by selecting $\gamma_{2}$ and $\gamma_{3}$ small enough to suppress the amplitude of $\frac{1}{2} \frac{\partial f_{2}\left(r_{3}, r_{4}\right)}{\partial r_{4}}$ and $\frac{d_{3} k_{3} \sigma r_{2}^{2}}{2 d_{2} k_{2}}\left(c_{2}|w|+\omega^{0}\right)$ relative to $\delta_{2}$. The difference appears in $q_{3}^{\prime}$ where $c_{3}$ now must be used to guarantee a positive $q_{3}^{\prime}$. Choosing $\frac{d_{2}}{d_{3}}$ sufficiently small will suppress the amplitude of $\frac{k_{2}}{2 k_{3} \gamma_{2}} \frac{\partial f_{2}\left(r_{3}, r_{4}\right)}{\partial r_{4}}$, but since $\gamma_{3}$ already is chosen $c_{3}$ must be sufficiently large relative to $\frac{\sigma r_{2}^{2}}{2 \gamma_{3}}\left(c_{2}|w|+\omega^{0}\right)$. The analysis is summarized in a proposition.

Proposition 4: Assume that $\frac{\partial p_{2}(w, \omega)}{\partial \omega}$ is bounded for bounded $(w, \omega)$ and $f_{1}\left(x_{1}\right)$ satisfy $(16)$. Then the control law

$$
u=-\frac{c_{2} k_{2}}{k_{3}} f_{2}\left(x_{2}, x_{3}\right)+\frac{c_{2} k_{2}}{k_{3}} x_{1}-c_{2} c_{3} x_{2}-c_{3} x_{3}
$$

with

$$
c_{2} \geq\left.\frac{\frac{\partial f_{2}\left(r_{1}, r_{2}\right)}{\partial r_{1}}+\delta_{2}}{\frac{\partial f_{2}\left(r_{1}, r_{2}\right)}{\partial r_{2}}}\right|_{\left(r_{1}, r_{2}\right)=\left(x_{2},-c_{2} x_{2}\right)}
$$

and $c_{3}>0$ sufficiently large, makes the origin of (9)(11) asymptotically stable. Moreover, the region of attraction increases with increasing amplitude of $c_{3}$ and semi global asymptotic stability is achieved by letting $c_{3}$ approach infinity.

Following the arguments of Corollary 3 , a similar corollary can be made for Proposition 4.

Corollary 5: The control law from Proposition 4 makes the origin of (9)-(11) exponentially stable. Moreover, the region of attraction increases with increasing amplitude of $c_{3}$ and semi global exponential stability is achieved by letting $c_{3}$ approach infinity.

Also here the only difference of the proposition and the corollary lies in the explicit use of (4) in the corollary rather than the property (16).

\section{Simulation}

The compressor map used in simulations is the same as used in [11]. This is a map based on measurement data for which third order approximations in both compressor speed and mass flow is done to make it continuos in these variables. The upper left plot in Fig. 3 shows a third order approximations of speed lines based on measurements, whereas the upper right plot of Fig. 3 shows a comparison of these lines with the resulting approximated compressor map.

Using (6) and (7) the valve characteristics can be plotted in the compressor map. This is illustrated in the lower left plot of Fig. 3 for two different throttle openings, where the intersection of compressor and throttle characteristics constitutes the possible equilibrium of the system using only the drive torque as input. This means that freedom to choose a desired operating point for the compression system using only the drive torque as actuator is limited by the valve characteristics.

Using (7) and (8) the torque characteristic can be plotted in the compressor map. This is illustrated in the lower right plot in Fig. 3 for two constant torque inputs of different amplitude. Also shown in this plot are the two different throttle openings, where the intersection of throttle and torque characteristic will give system equilibrium for the corresponding valve opening and compressor torque.
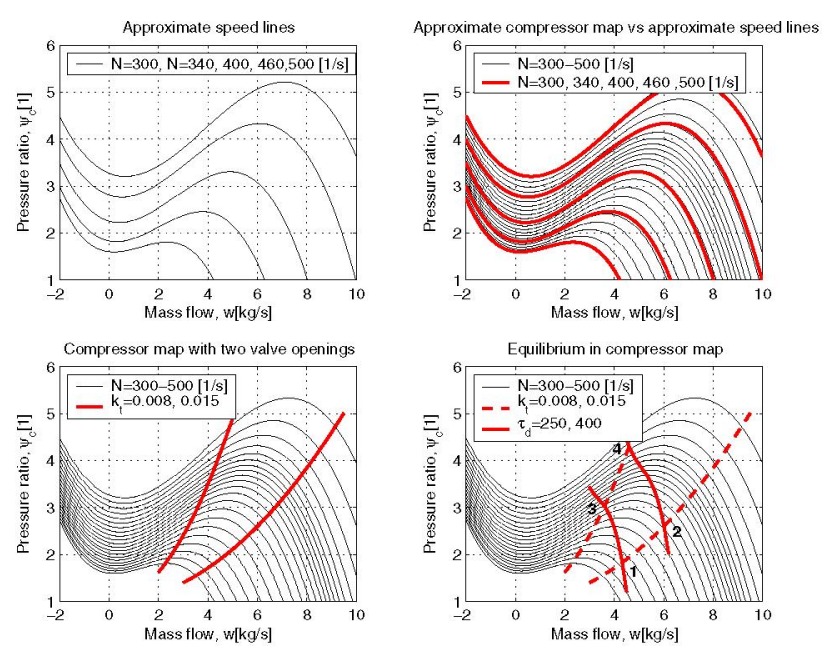

Fig. 3. Compressor map and system equilibrium

The simulation will go through a scenario in which the system is initially operating in point 1 of of the lower right plot in Fig. $1\left(k_{t}=0.015\right.$ and $\left.\tau_{d}=250\right)$. Next the system is driven to operating point $2\left(k_{t}=0.015\right.$ and $\left.\tau_{d}=400\right)$, which involves a change of torque input for the uncontrolled system and a change of desired equilibrium for the controlled system. Finally the system is driven to operating point 4 ( $k_{t}=0.008$ and $\tau_{d}=400$ ), which involves a change in the throttle opening for the both uncontrolled and controlled system in addition to a change of desired equilibrium for the controlled system. All changes of system parameters done to change operating points are filtered through a fist order linear filter. This is done to get a realistic response from the control law with respect to commanded torque. Without this filter the system still poses the same qualitative response as with the filter, but the commanded torque becomes unreasonably large in the transients.

For the scenario described, the throttle openings constitutes equilibrium points for which the compressor characteristics has a negative and positive slope in $w$ respectively. From the literature it is well known that a negative slope constitutes stable equilibriums. For positive slope however, the system need not be stable and surge can occur.

The simulation result is shown in Fig. 4 for the open loop and the control law from Proposition 2 in the left and right column respectively.

The open loop response shows stable behavior in the first two operating points before eventually entering surge in the last operating point. From the plot of compressor speed, it seems like this state is not oscillating. However, a closer examination reveals relative small oscillation also for this state. The closed loop response shows stable behavior in all operating points. Moreover, convergence to desired equilibrium is faster than that of the open loop. Especially in the transition to the last operating point it can be seen to converge faster. 

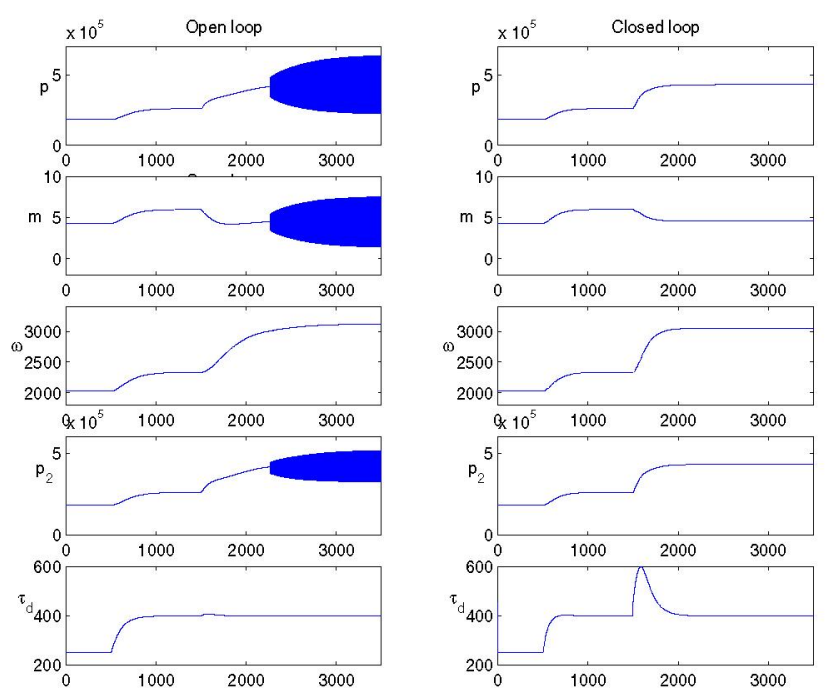

Fig. 4. Simulation of open and closed loop

\section{CONCluding REMARKS}

The proposed control laws require measurement of plenum pressure, mass flow and compressor velocity. In addition to these measurements, information of pressure downstream the compressor and compressor torque are required. For pressure downstream the compressor a model or a measurement can be used. In many cases a measurement is preferable since the compressor model may be poorly known, especially to the left of the surge line. For the compressor torque a measurement, model or information of compressor torque in equilibrium is required.

Writing the proposed control laws in terms of its original variables and desired equilibrium results in

$$
\begin{aligned}
\tau_{d}^{(1)}= & \tau_{c}(w, \omega)-\frac{c_{2} k_{2}}{k_{3}}\left(p_{2}(w, \omega)-p\right)-c_{2} c_{3}\left(w-w^{0}\right) \\
& -c_{3}\left(\omega-\omega^{0}\right) \\
\tau_{d}^{(2)}= & \tau_{d}^{0}-\frac{c_{2} k_{2}}{k_{3}}\left(p_{2}(w, \omega)-p\right)-c_{2} c_{3}\left(w-w^{0}\right) \\
& -c_{3}\left(\omega-\omega^{0}\right)
\end{aligned}
$$

where the only difference lies in the compressor torque terms, both of which can be considered as the offset input required in equilibrium since $\tau_{c}(w, \omega)$ approaches $\tau_{d}^{0}$ as the system approaches equilibrium. Using $\tau_{d}^{0}$ will cause problems for step changes in desired equilibrium since it will require a step change in the actuator, whereas the requirement imposed by $\tau_{c}(w, \omega)$ on actuator response will be limited by the convergence rates of $w$ and $\omega$. On the other hand, using $\tau_{c}(w, \omega)$ requires explicit knowledge of compressor torque, either measurement or model, whereas $\tau_{d}^{0}$ only requires knowledge of compressor torque in equilibrium. The terms $w-w^{0}$ and $w-w^{0}$ can be considered as contributors for convergence towards desired equilibrium since they contain explicit knowledge of this point, as well as anti surge contributors since surging will involve deviation from equilibrium. The term $p_{2}(w, \omega)-p$ can be considered as anti surge contributor, assuming these pressures do not cancel each other out in surge. The term can also be considered as an implicit contributor for $p$ converging toward a desired equilibrium since $p$ equals $p_{2}(w, \omega)$ in equilibrium.

Neither Proposition 2 nor Proposition 4 makes explicit use of a throttle valve model, they only require it to be strictly passive in $p$. This implies that any application in which the termination of this system is strictly passive in $p$ is asymptotically stable with the proposed control laws .

As pointed out in the simulation section, using only the drive torque as control input leaves little freedom in choosing the desired equilibrium. In view of the control law we are limited to choose either $w^{0}$ or $\omega^{0}$ and calculate the other. This calculation involves using a model for the compressor characteristics, which in many cases are poorly known. One solution to this problem could be to estimate one of the equilibrium states in question. This is also an approach that would be interesting with respect to the constant $\tau_{d}^{0}$ due to its dependence on $w^{0}$ and $\omega^{0}$.

The control law makes explicit use of the model parameters $a_{01}, V_{p}, A_{1}$ and $L_{c}$ for which uncertainty can be an issue. One approach towards a solution of this problem would be to estimate these constants. However, they only appear in one configuration, $\frac{a_{01}^{2} L_{c}}{A_{1} V_{p}}$, which suggest that the complexity of this problem is identical to that of one unknown constant.

Measuring mass flow is both expensive and hampered with high noise levels. This motivates the use of estimated mass flow in the control law rather than a measurement. One such observer is presented in [12], where it is shown that by using this observer together with the control laws from Corollary 3 or Corollary 5 , the stability results do not change.

\section{REFERENCES}

[1] A. H. Epstein, J. E. Ffowes Williams, and E. M. Greitzer, "Active supression of aerodynamic instabilities in turbomachines," Journal of Propulsion and Power, vol. 5, no. 2, pp. 204-211, 1989.

[2] J. T. Gravdahl and O. Egeland, Compressor surge and rotating stall: modeling and control, ser. Advances in Industrial Control. SpringerVerlag, 1999.

[3] F. Willems and B. de Jager, "Modeling and control of compressor flow instabilities," Control Systems Magazine, vol. 19, no. 5, pp. 818, October 1999.

[4] J. T. Gravdahl, O. Egeland, and S. O. Vatland, "Drive torque actuation in active surge control of centrifugal compressors," Automatica, vol. 38, no. 11, pp. 1881-1893, 2002.

[5] E. M. Greitzer, "Surge and rotating stall in axial flow compressors, part i: Theoretical compression system model," Journal of Engineering for Power, vol. 98, pp. 190-198, 1976.

[6] D. A. Fink, N. A. Cumpsty, and E. M. Greitzer, "Surge dynamics in free-spool centrifugal compressor system," Journal of Turbomachinery, vol. 114, pp. 321-332, 1992.

[7] J. T. Gravdahl, F. Willems, B. de Jager, and O. Egeland, "Modeling of surge in variable speed centrifugal compressors: Experimental validation," ALAA Journal of Propulsion and Power, vol. 20, no. 5, pp. 849-857, 2004.

[8] M. Krstic, I. Kanellakopoulos, and P. Kokotovic, Nonlinear and Adaptive Control Design. John Wiley \& Sons, 1995.

[9] M. Krstic, D. Fontaine, and P. Kokotovic, "Useful nonlinearities and global stabilization of bifurcations in a model of jet engine surge and stall," IEEE Transactions on Automatic Control, vol. 43, no. 12, pp. 1739-1745, December 1998.

[10] H. K. Khalil, Nonlinear System, 3rd ed. Prentice-Hall, 2002.

[11] J. T. Gravdahl, O. Egeland, and S. O. Vatland, "Active surge control of centrifugal compressors using drive torque," in Procedings of the 40th IEEE Conference on Desition and Control, December 2001.

[12] B. Bøhagen and J. T. Gravdahl, "A GES mass flow observer for compression systems: Design and experiments," in Proceedings of the American Control Conference, 2004, pp. 1528-1533. 